\title{
The distribution of genetic diversity in the Neofusicoccum parvum/N. ribis complex suggests structure correlated with level of disturbance
}

\author{
Draginja Pavlic-Zupanc ${ }^{a, d, *}$, Michael J. Wingfield ${ }^{a}$, Emilie Boissin $^{c}$, Bernard Slippers $^{b}$
}

\author{
${ }^{a}$ Department of Microbiology and Plant Pathology, Centre of Excellence in Tree Health Biotechnology, Forestry and \\ Agricultural Biotechnology Institute (FABI), Faculty of Natural and Agricultural Sciences, University of Pretoria, \\ Pretoria 0002, South Africa \\ ${ }^{\mathrm{b}}$ Department of Genetics, Centre of Excellence in Tree Health Biotechnology, Forestry and Agricultural Biotechnology \\ Institute (FABI), Faculty of Natural and Agricultural Sciences, University of Pretoria, Pretoria 0002, South Africa \\ 'USR3278-CRIOBE-CNRS-EPHE, Laboratoire d'excellence "CORAIL”, Université de Perpignan-CBETM, \\ 58 Rue Paul Alduy, 66860 Perpignan CEDEX, France \\ diosystematics Programme, Plant Protection Research Institute, Agricultural Research Council (ARC-PPRI), \\ Private Bag X134, Pretoria 0121, South Africa
}

\begin{abstract}
A b s t r a c t
Plants and animals adapted to colonize disturbed sites might also be better invaders, but this phenomenon has not been widely considered in fungi. We investigated genetic diversity and structure amongst isolates of Neofusicoccum parvum, N. cordaticola, N. kwam-bonambiense and N. umdonicola that coexist sympatrically on a native tree, Syzygium cor-datum, across its distribution in South Africa. Species composition varied among stands, with dominance of $N$. parvum in disturbed stands, and absence in undisturbed stands, where the other species dominated. N. parvum populations from trees planted in urban environments were more genetically diverse than populations from human disturbed stands of $\mathrm{S}$. cordatum. Bayesian analysis clustered $\mathrm{N}$. parvum isolates in three sub-populations, suggesting three sources of origin. These results support the hypothesis that as a generalist N. parvum will dominate human disturbed sites and trees in urban areas, indicating strong potential for invasion, and its spreading from non-native hosts to native $S$. cordatum, rather than vice versa.
\end{abstract}

Keywords:

Anthropogenic disturbance

Biogeography

Botryosphaeriaceae

Disturbed habitats

Diversity

Invasion ecology

Native trees

\footnotetext{
* Corresponding author. Biosystematics Programme-Mycology Unit, Plant Protection Research Institute, Agricultural Research Council (ARC-PPRI), Private Bag X134, Pretoria 0121, South Africa. Tel.: +27 12808 8289; fax: +27 128088297.

E-mail addresses: pavlicdr@gmail.com, pavlicd@arc.agric.za (D. Pavlic-Zupanc).
} 


\section{Introduction}

Organisms with broad niche breadths are often prominent colonizers of disturbed environments in their home range but invaders in new regions (Lee and Gelembiuk, 2008). This is also true for some invading fungi (Fisher et al., 2012; Santini et al., 2013), although the phenomenon has not been widely studied in this group of organisms. Slippers and Wingfield (2007) noticed that a number of the prominent tree pathogenic fungi in the Botryosphaeriaceae are generalists in terms of host associations. Many of these species also appear to be widely distributed, most likely invasive in many regions, and occur in human associated, disturbed environments. In this study, we thus question whether some of these generalist species might act as "weeds", colonizing disturbed environments more effectively than native environments that are presumably more ecologically diverse and stable.

The fungal Neofusicoccum parvum/N. ribis species complex (Botryosphaeriaceae, Ascomycetes) (Crous et al., 2006) was thought to include two sister species that are fungal plant pathogens on a variety of woody hosts, most frequently reported from the Southern Hemisphere (Slippers and Wingfield, 2007). Recent studies of this complex on a native South African tree, Syzygium cordatum, resulted in the identification of three additional cryptic species using a Genealogical Concordance Phylogenetic Species Recognition (GCPSR) approach based on concordance of multiple locus sequence data (Pavlic et al., 2009a,b). These were described as the phylogenetic, cryptic species N. cordaticola, N. kwambonambiense and N. umdonicola (Pavlic et al., 2009b). Subsequently, additional cryptic species were identified in the $\mathrm{N}$. parvum/N. ribis complex, including N. batangarum (Begoude et al., 2010), N. occulatum and Neofusicoccum sp. karanda (Sakalidis et al., 2011).

The reproductive cycle of the haploid Neofusicoccum species in the N. parvum/N. ribis complex is underexplored, and mating types of these species are not known. Neofusicoccum parvum and $N$. ribis are known to produce both asexual and sexual structures under field conditions, but are most commonly encountered in their asexual spore (conidial) stage (Pennycook and Samuels, 1985; Slippers et al., 2004a; Pavlic et al., 2007). Other species in the complex are known only from their asexual stage.

The global distribution and host association of members of the N. parvum/N. ribis complex has recently been characterized (Sakalidis et al., 2013). That study confirmed the widespread and generalist pathogen status of N. parvum, which was shown to occur on 90 hosts across six continents. On the contrary, $N$. ribis was identified only from a single host species, Ribes sp. in the USA, from which it was originally described. The other cryptic species in this complex were more widespread than $N$. ribis, but they had more restricted geographical distributions and host associations than N. parvum (Sakalidis et al., 2013). The cryptic species in the N. paruum/N. ribis complex have been recorded as pathogens on cultivated, economically important non-native fruit and forestry trees (Slippers et al., 2004b, 2007; Mohali et al., 2007) and also on forest trees in native ecosystems (Slippers et al., 2005; Burgess et al., 2005; Pavlic et al., 2007). They are commonly isolated as endophytes that reside, asymptomatically, within plant tissues of numerous woody hosts (Slippers and Wingfield, 2007). This makes them ideal candidates for undetected, long distance dispersal by humans, together with plant germplasm traded for agriculture and forestry.

Syzygium cordatum is an evergreen broad-leafed tree that belongs to the Southern Hemisphere family Myrtaceae (Palgrave, 1977), and is the most widely distributed Syzygium species in South Africa. Naturally seeded S. cordatum trees occur widely along the eastern part of the country as single trees or patches of trees remaining within human disturbed areas, e.g. amongst stands of non-native Eucalypts plantations, or in undisturbed stands (e.g. Nature Reserves). Syzygium cordatum is also planted as ornamental in urban environments across the country. The distribution of S. cordatum across these three different disturbance types (undisturbed, human disturbed, and in cities), and infection of four cryptic species from N. paruum/N. ribis complex, make this an interesting model to better understand the influence of disturbance on the diversity and distribution of these fungi.

In this study, we used previously designed SSR markers (Slippers et al., 2004a) to: (i) assess genetic diversity and structure of four species, N. parvum, N. cordaticola, N. kwambonambiense and N. umdonicola that coexist sympatrically on $\mathrm{S}$. cordatum trees in South Africa, and (ii) analyze the distribution of the species and population diversity across three habitat types: undisturbed stands of S. cordatum (e.g. Nature Reserve), human disturbed stands (e.g. naturally regenerated S. cordatum trees remaining within non-native Eucalyptus plantations) and planted S. cordatum as ornamentals in urban areas.

\section{Materials and methods}

\section{Fungal isolates}

The isolates used in this study were collected during a survey of the Botryosphaeriaceae on native S. cordatum trees in different geographical locations of South Africa, between Feb. 2001 and Mar. 2003 (Supplementary Table 1, Fig 1). Syzygium cordatum trees do not grow in persistent forests, but rather in patches or as solitary trees and thus all available trees in 13 sampling sites were examined. Between 1 and 45 trees were examined from each site. The isolates were obtained from 1 to 17 trees per site and 1-5 isolates were collected per tree (Supplementary Table 1). From each tree, isolations were made from twigs showing die-back symptoms, and asymptomatic twig, leaf and fruit as described by Pavlic et al. (2007, 2009a,b). Of the 114 isolates used in this study, 50 were collected from undisturbed habitats of S. cordatum (five sites), 31 were collected from human-disturbed habitats (three sites) and 33 isolates were collected from planted S. cordatum trees in urban areas (five sites) (Supplementary Table 1, Table 1; Fig 1). One hundred and two isolates were identified in previous studies, and additional 12 (isolated from asymptomatic twigs) in this study, as species in the N. parvum/N. ribis complex including $N$. parvum (48 isolates), N. cordaticola (17), N. kwambonambiense (14) and N. umdonicola (35), based on DNA sequence data for at least one locus and PCR-RFLP analysis 


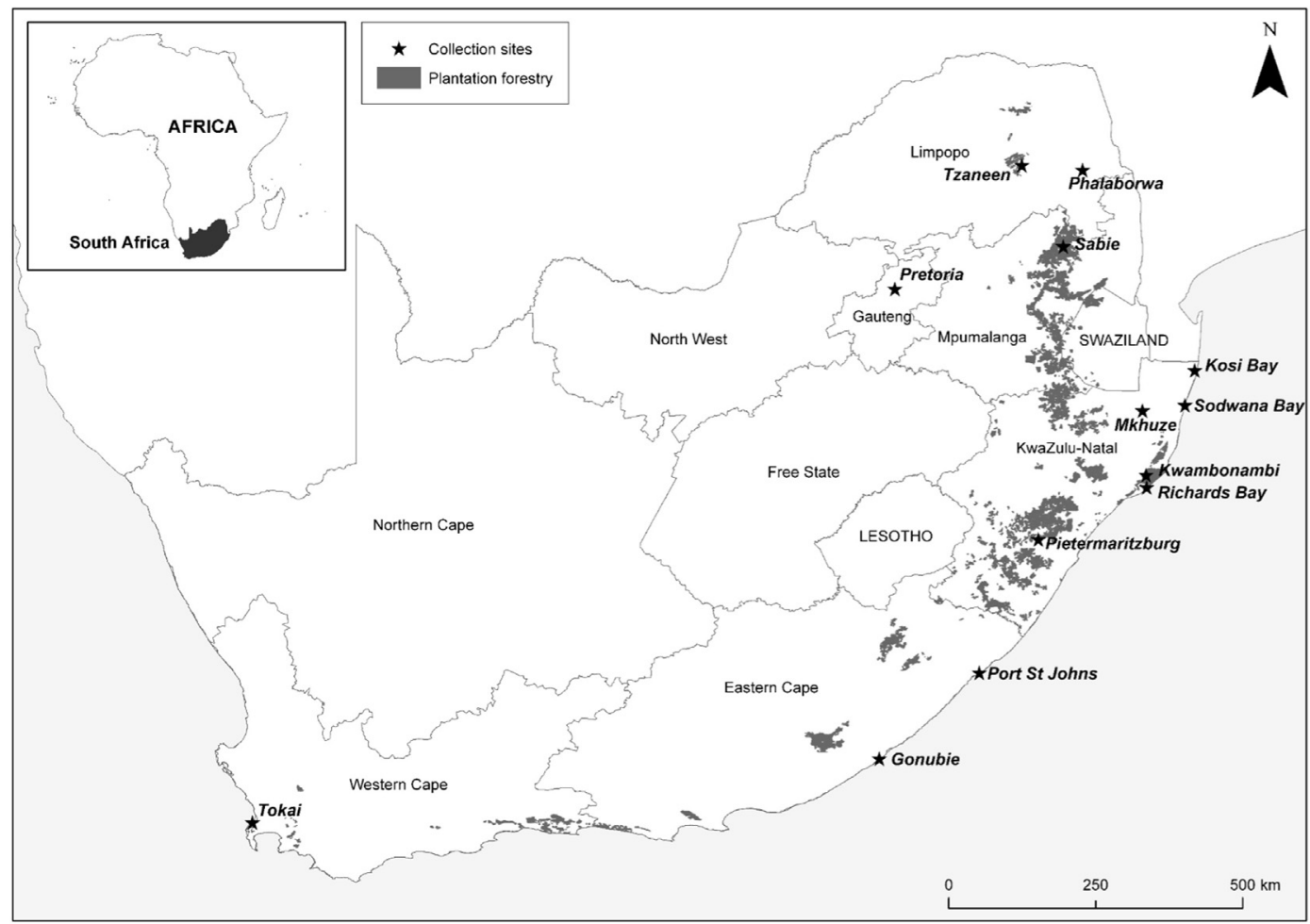

Fig 1 - Map of South Africa showing collection sites and plantation forestry. Tzaneen (P), Palaborwa (P), Pretoria (P), Sabie (UD), Kosi Bay (UD), Mkhuze (UD), Sodwana Bay (UD), Kwambonambi (HD), Richards Bay (UD), Pietermaritzburg (P), Port St. Johns (HD), Gonubie (HD), Tokai (P). P = Isolates collected from planted trees in urban areas; DU = Isolates collected from trees in undisturbed stands; HD = Isolates collected from trees human disturbed stands.

(Pavlic et al., 2007, 2009a,b) (Supplementary Table 1). Isolates of all four species were collected from twigs showing die-back symptoms and asymptomatic, visually healthy tissues. All haploid, single conidial cultures used in this study are maintained in the culture collection (CMW) of the Forestry and Agricultural Biotechnology Institute (FABI), University of Pretoria, Pretoria, South Africa and representative isolates have been deposited in the collection of the Centraalbureau voor Schimmelcultures (CBS), Utrecht, The Netherlands.

\section{DNA extraction, microsatellite-PCR amplification and genotyping}

The haploid, single conidial cultures were grown on $2 \%$ malt extract agar (MEA) (20 g malt extract, $15 \mathrm{~g}$ agar; Biolab, Midrand, Johannesburg, S.A. and $1000 \mathrm{~mL}$ deionized water) for 7 days at $25{ }^{\circ} \mathrm{C}$ in the dark. DNA was extracted from the mycelium following the modified phenol-chloroform DNA extraction method described in Pavlic et al. (2007). DNA was separated by electrophoresis on $1.5 \%$ agarose gels, stained with ethidium bromide and visualized under ultraviolet light. DNA concentrations were estimated against $\lambda$ standard size markers. Seven loci that contained microsatellite sequences were amplified for all isolates, using fluorescently-labeled primer pairs designed for species of Botryosphaeriaceae with Fusicoccum-like anamorphs (Slippers et al., 2004a). PCR reactions were performed using an Eppendorf Mastercycler PERSONAL (Perkin-Elmer, Germany) and the following protocol: $94^{\circ} \mathrm{C}$ for $2 \mathrm{~min}$ initial denaturation; 40 cycles of $94^{\circ} \mathrm{C}$ for $30 \mathrm{~s}$, 55 or $62{ }^{\circ} \mathrm{C}$ for $30 \mathrm{~s}, 72{ }^{\circ} \mathrm{C}$ for $1 \mathrm{~min}$; and $72{ }^{\circ} \mathrm{C}$ for $7 \mathrm{~min}$ final extension. PCR products were separated in $2 \%$ agarose gels stained with ethidium bromide and visualized under UV light. Sizes of PCR products were estimated by comparison with a $100 \mathrm{bp}$ molecular weight marker (Fermentas Life Sciences). Allele sizes of labeled microsatellite-PCR products were determined on an ABI PRISM $3100^{\mathrm{TM}}$ automated DNA sequencer (Perkin-Elmer, Warrington, U.K.) and compared against a GENESCAN-500 LIZ (Perkin-Elmer Applied Biosystems, Warrington, U.K.) internal size standard. Because fragment sizes overlapped for some of the amplicons, two separate gels were run for each sample. Allele sizes were analyzed with GENESCAN 3.7 and GENOTYPER 3 software (Perkin-Elmer Applied Biosystems, Foster City, CA, USA).

\section{Microsatellite analyses}

The program POPGENE version 1.31 (Yeh et al., 1999) was used to calculate allele frequencies at each microsatellite locus for each of four populations representing four species identified in the N. paruum/N. ribis complex from S. cordatum. For each population, the observed number of alleles $\left(n_{\mathrm{a}}\right)$, number of private alleles, number and percentage of polymorphic loci $(\mathrm{P})$ were evaluated. The genetic diversity of each sample was estimated by calculating the unbiased expected heterozygosity (Hnb, Nei, 1978) in GenClone (Arnaud-Haond and Belkhir, 2007). Additionally, in GenClONE, a rarefaction 
Table 1 - Distribution of isolates of four Neofusicoccum spp. collected from S. cordatum trees in South Africa

\begin{tabular}{|c|c|c|c|c|}
\hline Collection sites ${ }^{a, b, c}$ & N. cordaticola & N. kwambonambiense & N. umdonicola & N. parvum \\
\hline Tzaneen (1) P & - & 2 & - & 10 \\
\hline Phalaborwa (2) P & - & - & - & 2 \\
\hline Pretoria (3) P & - & - & - & 2 \\
\hline Sabie (4) UD & 9 & 4 & - & - \\
\hline Kosi Bay (5) UD & 1 & - & 12 & - \\
\hline Mkhuze (6) UD & 1 & - & 2 & - \\
\hline Sodwana Bay (7) UD & 1 & - & 14 & - \\
\hline Kwambonambi (8) HD & 3 & 4 & 5 & 15 \\
\hline Richards Bay (9) UD & 2 & 4 & - & - \\
\hline Pietermaritzburg (10) P & - & - & - & 14 \\
\hline Port St. Johns (11) HD & - & - & 1 & 1 \\
\hline Gonubie (12) HD & - & - & 1 & 1 \\
\hline Tokai (13) P & - & - & - & 3 \\
\hline Total & 17 & 14 & 35 & 48 \\
\hline
\end{tabular}

method allows for comparison of heterozygosity among samples with different sample sizes. The unbiased expected heterozygosity was compared for a standard size of 10 isolates corresponding to the sample with the lowest number of isolates (population two of N. paruum). Allelic richness and Private allelic richness were computed using ADZE (Szpiech et al., 2008). The software also implements a rarefaction approach allowing for a comparison of the values for a standard size of 10 (corresponding to the smallest sample).

Linkage Disequilibrium between pairs of loci was investigated for each species using the Black and Krafsur (1985) algorithm available in GeNETIx v4.05 (Belkhir et al., 2004). The Isolation-by-Distance (IbD) hypothesis was tested using the Fst/(1 - Fst) genetic distance (Rousset, 1997) and the log geographic distances between locations. The analysis was conducted on the species represented by the largest number of samples: N. umdonicola and N. parvum, as well as population one of $N$. parvum alone using the Mantel test implemented in GenETIX.

The genetic relationship of populations and species was assessed using two different methods: (i) a principal coordinate analyses (PCoA) (in GenAlex v6.5, Peakall and Smouse, 2006, 2012) and (ii) Bayesian clustering (in STRUCtURE v2.3.4, Pritchard et al., 2000). The multivariate analysis (PCoA) is based on distances between multilocus genotypes of individuals and does not make any assumption on the underlying population genetic model. The PCoA was performed using genetic distances and the co-variance standardized method. STRUCTURE, on the other hand, attempts to minimize the Hardy-Weinberg Disequilibrium and Linkage Disequilibrium within clusters in order to find the most likely number of clusters in a data set.

This Bayesian clustering was used to determine whether isolates in the N. parvum/N. ribis complex could be subdivided into $\mathrm{K}$ genetically distinct groups. STRUCTURE assumes a model in which there are $K$ populations (where $K$ may be unknown), each of which is characterized by a set of allele frequencies at each locus. Individuals in the sample are probabilistically assigned to a single population, or jointly to two or more populations if their haplotypes indicate that they are admixed. To determine the most likely number of genetically distinct groups or clusters $(K)$ in the sample, 20 independent runs for each $\mathrm{K}=1$ to 10 were carried out at 100000 Markov chain Monte Carlo (MCMC) repetitions following a burn-in of 50000 iterations. The program was run assuming both admixture and no admixture among the populations and additional parameters assumed were: different values of $F_{\mathrm{ST}}$ for different subpopulations, prior mean of $\mathrm{F}_{\mathrm{ST}} 0.01$, standard deviation (SD) of $F_{\mathrm{ST}} 0.05$ and constant lambda value at 1 . The most probable number of clusters was obtained using the Evanno's approach (Evanno et al., 2005) implemented online in Structure harvester (Earl and VonHoldt, 2012). The analyses were run both on the full dataset (114 isolates) and on a clonecorrected data set (61 isolates) where only one of each of the haplotypes was included in the analyses.

Analysis of molecular variance (AMOVA) was carried out using Genalex. We examined the partitioning of genetic variation among and within the geographically defined populations and regions of $N$. umdonicola, N. parvum and sub-population one of $N$. parvum. The other species and sub-populations two and three of $N$. parvum were not included in this analysis because of small sample size. The analysis of molecular variance was performed on the full datasets.

\section{Results}

\section{Genetic structure and diversity}

For the entire dataset of 114 isolates that belong to N. parvum/ $N$. ribis complex from S. cordatum, a total of 61 alleles were observed across seven loci examined (Table 2). There were 26 alleles detected amongst the $17 \mathrm{~N}$. cordaticola individuals, 16 
Table 2 - Allele size (bp) and frequency at seven loci in four Neofusicoccum spp., N. cordaticola (N. c), N. kwambonambiense (N. k), N. umdonicola (N. u) and N. parvum collected from Syzygium cordatum in South Africa

\begin{tabular}{|c|c|c|c|c|c|c|c|c|}
\hline \multirow[t]{2}{*}{ Locus } & \multirow[t]{2}{*}{ Allele ${ }^{a}$} & \multirow[t]{2}{*}{ N. C } & \multirow[t]{2}{*}{ N. k } & \multirow[t]{2}{*}{ N. u } & \multicolumn{4}{|c|}{ N. parvum ${ }^{\mathrm{b}}$} \\
\hline & & & & & Pop 1 & Pop 2 & Pop 3 & All \\
\hline \multirow[t]{8}{*}{ BotF11 } & 420 & - & 1.000 & - & - & - & - & - \\
\hline & 427 & - & - & - & 0.111 & 0.200 & - & 0.104 \\
\hline & 428 & - & - & - & 0.852 & 0.800 & 0.909 & 0.854 \\
\hline & 431 & 0.471 & - & 0.400 & - & - & 0.091 & - \\
\hline & 432 & - & - & 0.543 & - & - & - & 0.021 \\
\hline & 433 & - & - & 0.057 & - & - & - & - \\
\hline & 435 & 0.471 & - & - & - & - & - & - \\
\hline & null & 0.058 & - & - & 0.037 & - & - & 0.021 \\
\hline \multirow[t]{8}{*}{ BotF15 } & 365 & - & - & - & - & 0.900 & - & 0.187 \\
\hline & 374 & - & - & - & - & 0.100 & - & 0.021 \\
\hline & 377 & 1.000 & - & 1.000 & 0.963 & - & 0.454 & 0.646 \\
\hline & 378 & - & - & - & 0.037 & - & - & 0.021 \\
\hline & 389 & - & - & - & - & - & 0.364 & 0.083 \\
\hline & 390 & - & - & - & - & - & 0.182 & 0.042 \\
\hline & 395 & - & 0.857 & - & - & - & - & - \\
\hline & 396 & - & 0.143 & - & - & - & - & - \\
\hline \multirow[t]{9}{*}{ BotF17 } & 229 & - & 1.000 & 0.971 & - & - & - & - \\
\hline & 233 & 0.117 & - & - & - & - & 0.455 & 0.104 \\
\hline & 234 & 0.882 & - & - & - & - & - & - \\
\hline & 244 & - & - & - & - & - & 0.455 & 0.104 \\
\hline & 246 & - & - & - & 0.148 & - & - & 0.083 \\
\hline & 247 & - & - & - & - & - & 0.090 & 0.021 \\
\hline & 249 & - & - & 0.029 & 0.852 & - & - & 0.479 \\
\hline & 256 & - & - & - & - & 0.500 & - & 0.104 \\
\hline & 259 & - & - & - & - & 0.500 & - & 0.104 \\
\hline \multirow[t]{10}{*}{ BotF21 } & 203 & - & 0.071 & 0.200 & - & - & - & - \\
\hline & 204 & 0.176 & 0.143 & 0.743 & 0.111 & - & - & 0.062 \\
\hline & 206 & - & - & - & - & - & 0.090 & 0.021 \\
\hline & 207 & 0.118 & - & - & 0.074 & 0.400 & 0.273 & 0.187 \\
\hline & 208 & 0.118 & 0.143 & - & - & - & 0.455 & 0.104 \\
\hline & 209 & 0.059 & 0.071 & - & - & 0.600 & - & 0.125 \\
\hline & 219 & 0.294 & 0.429 & - & 0.815 & - & 0.182 & 0.500 \\
\hline & 229 & - & 0.071 & 0.057 & - & - & - & - \\
\hline & 234 & 0.176 & - & - & - & - & - & - \\
\hline & null & 0.059 & 0.071 & - & - & - & - & - \\
\hline \multirow[t]{8}{*}{ BotF23 } & 422 & - & - & 0.914 & 1.000 & - & - & 0.562 \\
\hline & 423 & - & - & - & - & - & 0.090 & 0.021 \\
\hline & 424 & - & - & - & - & - & 0.182 & 0.042 \\
\hline & 425 & - & - & - & - & 1.000 & 0.546 & 0.333 \\
\hline & 426 & 0.059 & 0.286 & - & - & - & 0.182 & 0.042 \\
\hline & 427 & 0.059 & 0.714 & - & - & - & - & - \\
\hline & 428 & 0.882 & - & 0.057 & - & - & - & - \\
\hline & null & - & - & 0.029 & - & - & - & - \\
\hline \multirow[t]{11}{*}{ BotF35 } & 222 & - & - & - & 0.148 & - & - & 0.083 \\
\hline & 225 & 0.059 & - & - & 0.852 & - & - & 0.480 \\
\hline & 238 & - & - & 0.800 & - & - & 0.546 & 0.125 \\
\hline & 239 & - & - & - & - & - & 0.364 & 0.083 \\
\hline & 244 & 0.529 & - & - & - & 0.900 & - & 0.187 \\
\hline & 245 & - & - & 0.200 & - & 0.100 & - & 0.021 \\
\hline & 247 & 0.294 & - & - & - & - & - & - \\
\hline & 253 & 0.059 & 0.429 & - & - & - & - & - \\
\hline & 255 & - & 0.571 & - & - & - & - & - \\
\hline & 265 & - & - & - & - & - & 0.090 & 0.021 \\
\hline & null & 0.059 & - & - & - & - & - & - \\
\hline \multirow[t]{7}{*}{ BotF37 } & 303 & 0.412 & - & - & - & - & - & - \\
\hline & 306 & 0.412 & - & - & - & - & - & - \\
\hline & 312 & - & - & 1.000 & 0.963 & - & - & 0.542 \\
\hline & 313 & 0.059 & - & - & 0.037 & 1.000 & 0.818 & 0.417 \\
\hline & 314 & - & - & - & - & - & 0.182 & 0.042 \\
\hline & 320 & 0.059 & 1.000 & - & - & - & - & - \\
\hline & null & 0.059 & - & - & - & - & - & - \\
\hline
\end{tabular}




\begin{tabular}{|c|c|c|c|c|c|c|c|}
\hline \multirow[t]{2}{*}{ Allele ${ }^{a}$} & \multirow[t]{2}{*}{ N. C } & \multirow[t]{2}{*}{ N. k } & \multirow[t]{2}{*}{ N. u } & \multicolumn{4}{|c|}{ N. parvum ${ }^{b}$} \\
\hline & & & & Pop 1 & Pop 2 & Pop 3 & All \\
\hline $\mathrm{N}$ & 17 & 14 & 35 & 27 & 10 & 11 & 48 \\
\hline$n_{\mathrm{a}}$ & 26 & 16 & 15 & 15 & 13 & 21 & 38 \\
\hline Allelic richness & $3.12(0.63)$ & $2.14(0.67)$ & $1.99(0.31)$ & $2.13(0.26)$ & $1.71(0.18)$ & $3.00(0.31)$ & $5.40(0.71)$ \\
\hline Allelic richness standardized for 10 isolates & $2.69(0.53)$ & $2.02(0.56)$ & $1.66(0.24)$ & $1.70(0.18)$ & $1.71(0.18)$ & $2.93(0.30)$ & $3.40(0.40)$ \\
\hline No. of private alleles & 8 & 4 & 2 & 3 & 4 & 10 & 20 \\
\hline Private allelic richness standardized for 10 isolates & $0.99(0.24)$ & $0.84(0.25)$ & $0.37(0.20)$ & $0.49(0.28)$ & $0.62(0.36)$ & $1.40(0.30)$ & $1.78(0.43)$ \\
\hline $\begin{array}{l}\mathrm{N}=\text { number of isolates. } \\
n_{\mathrm{a}}=\text { observed number of alleles. } \\
\text { a Alleles in bold are unique for each species. } \\
\text { b Populations one, two and three as defined in STR }\end{array}$ & (2) & . & 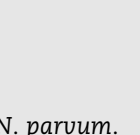 & & & & \\
\hline
\end{tabular}

alleles amongst the $14 \mathrm{~N}$. kwambonambiense individuals, 15 alleles amongst the $35 \mathrm{~N}$. umdonicola individuals and 38 alleles in the N. paruum populations (Table 2). Only one allele was shared among all four species. Private alleles were identified in each of these groups: twenty private alleles in N. parvum, eight in N. cordaticola, four in N. kwambonambiense, and two in N. umdonicola (Table 2). Population three of N. parvum displayed the highest private allelic richness (1.40) and N. umdonicola the lowest (0.37).

The mean total gene diversity across all isolates was the highest in $\mathrm{N}$. parvum $(\mathrm{Hnb}=0.579)$. Moderate gene diversity was observed in $\mathrm{N}$. cordaticola $(\mathrm{Hnb}=0.451)$ and low gene diversity in $\mathrm{N}$. kwambonambiense $(\mathrm{Hnb}=0.281)$ and $\mathrm{N}$. umdonicola populations $(\mathrm{Hnb}=0.214)$. The trends were similar when a correction for sample size was applied. All four species displayed significant linkage disequilibrium for all the pairwise comparisons (data not shown).

Six clusters were identified using STRUCTURE analyses with no prior population knowledge assumed (Fig 2A, B). Results were similar when using the entire dataset or the clonecorrected dataset, and using a model allowing admixture or not. Fig 2 presents the clustering on the clone-corrected dataset using an admixture model. The identified clusters supported the species distinctions recognized by Pavlic et al. (2009a,b) based on multiple gene sequence genealogies, as shown by the peak at $\mathrm{K}=4$ (Fig 2B), and they distinguished added subdivision in $N$. paruum, as shown by the peak at $K=6$ (Fig 2B). Isolates of each of the three species, $N$. cordaticola, $N$. kwambonambiense and N. umdonicola, were grouped in three different clusters, showing no further substructure within populations of these species. The three remaining clusters contained only $N$. parvum isolates (Fig 2A) representing a subdivision of this species into three sub-populations. The PCoA analyses showed the same clustering that separated the four species (Fig 3A, B). Further separation of N. parvum isolates into three subgroups was also supported in the PCoA analyses (Fig 3C).

\section{Genotypic diversity of N. parvum, N. parvum sub- population one and N. umdonicola}

Twenty-three genotypes were observed among 48 isolates of N. parvum from S. cordatum in South Africa (Supplementary Fig 1A). The three geographically defined populations of $N$. parvum encompassed a total of 41 isolates represented by 17 genotypes, four of which were from the Kwambonambi (KWM), nine from the Pietermaritzburg (PTM) and eight from the Tzaneen (TZ) populations (Supplementary Fig 1B-D). These three geographically defined populations do not correspond to the three genetically distinct groups (sub-populations) identified in the Bayesian analyses. The genotypic diversity of the N. parvum populations was higher for populations PTM $(\mathrm{Hnb}=0.532)$ and $\mathrm{TZ}(\mathrm{Hnb}=0.517)$ than KWM $(\mathrm{Hnb}=0.066)$. The low diversity in the KWM population was due to the predominance of a single genotype (S14), which accounted for $67 \%$ of the isolates collected in the Kwambonambi area (10 of 15 isolates). Genotype S14 was the only genotype shared between populations of $\mathrm{N}$. paruum from Kwambonambi and Pietermaritzburg, and three genotypes (S9, S11 and S12) were shared between the PTM and TZ populations. There were no genotypes shared between KWM and $\mathrm{TZ}$ populations.

From the AMOVA analysis that was applied to N. parvum dataset, the highest fraction of genetic variability (96\%) was within the populations and only $4 \%$ among the populations. Similarly, in the N. parvum sub-population one dataset, the highest fraction of variability was within populations (69\%) and $31 \%(p=0.011)$ of variability was among the geographic populations. When clustering specimens from Kwambonambi and Pietermaritzburg together in a region and against two other regions made up of specimens from (i) Tzaneen and (ii) Port Saint John's and Gonubie, the 'among regions' variance was $43 \%$ and significant ( $p=0.022$ ), the 'among populations within regions' variance was $5 \%$ (not significant, 'ns') and the 'within populations' variance was $52 \%$ $(p=0.015)$.

AMOVA analysis of $\mathrm{N}$. umdonicola populations showed that the 'among populations' variance was $9 \%$ (ns) and the 'within populations' variance was $91 \%$. When grouping Kosi Bay, Mkhuze and Sodwana Bay in a region and against two other regions made up of specimens from (i) Kwambonambi and (ii) Port Saint John's and Gonubie, the 'among regions' variance was $32 \%$, 'among populations' variance was $0 \%$ and the 'within populations' variance was $68 \%$, although none of these were statistically significant. The IbD tests were not significant for N. umdonicola, N. parvum and subpopulation one of $N$. parvum ( $p=0.27, p=0.45, p=0.10$ respectively). 

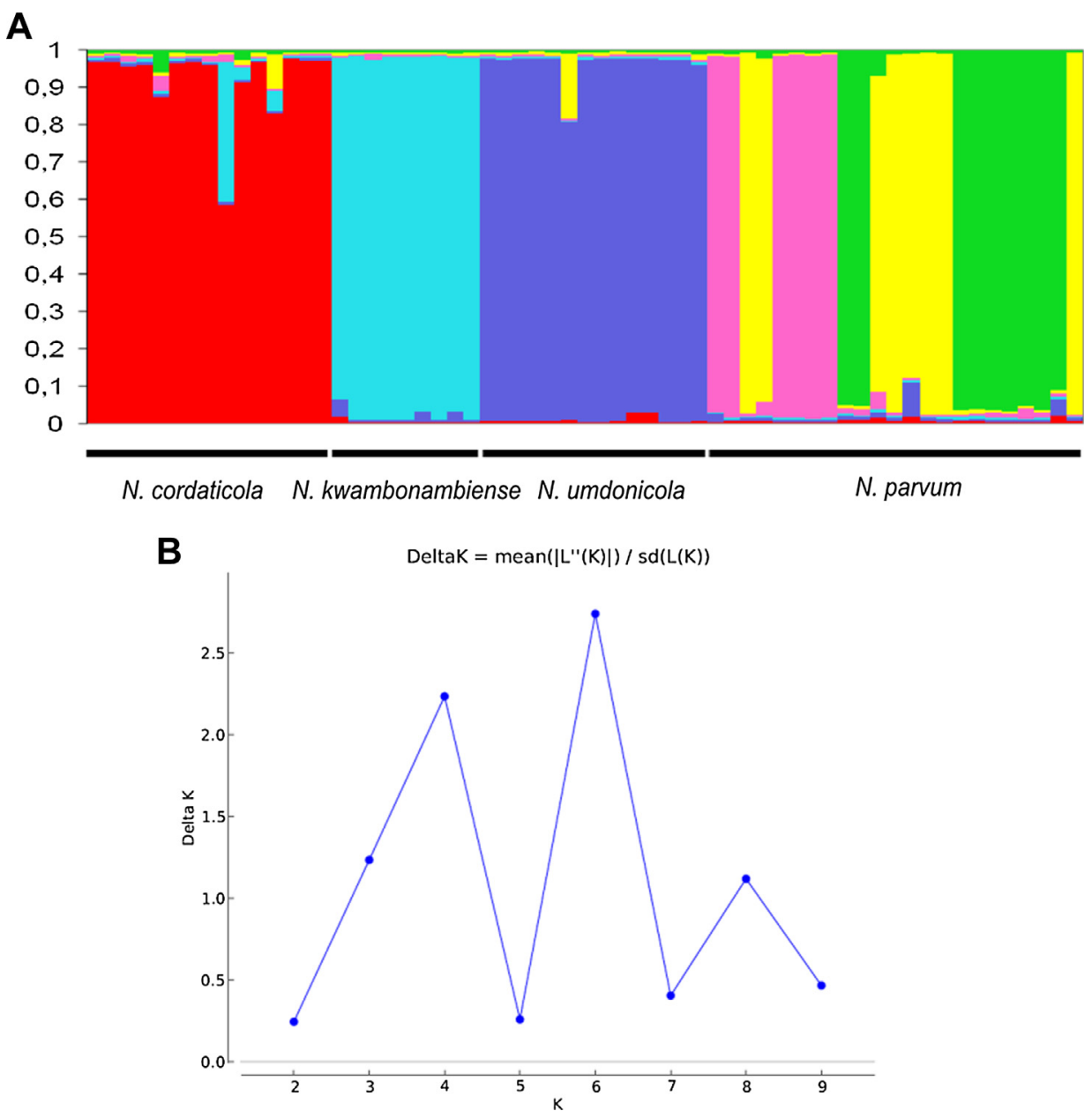

Fig 2 - The clustering outcome from structure analyses of the clone-corrected dataset of all isolates at $\mathrm{K}=6$ (A). Clusters are related to each of four Neofusicoccum species, $N$. cordaticola, $N$. kwambonambiense, $N$. umdonicola and $N$. parvum as indicated underneath the outcome. Note that isolates of $N$. parvum are distributed in three clusters indicated by pink, yellow and green. These clusters do not correspond to geographically defined groups of N. parvum; Evanno's graph representing the most likely number of clusters (K) (B).

\section{Distribution of Neofusicoccum spp. among habitat types}

The distribution of the four Neofusicoccum species on S. cordatum varied for the thirteen collection sites (Fig 4). Neofusicoccum parvum was isolated from trees in urban areas and humandisturbed sites, and was not found in undisturbed areas. Overall, N. parvum was the dominant Neofusicoccum species on S. cordatum in South Africa, making up $42 \%$ of all isolates.

\section{Discussion}

The species distribution pattern emerging from this study shows N. paruum as dominant and most abundant in habitats disturbed by or in close contact with human activities. This is in contrast to the three other cryptic species in the N. parvum/N. ribis complex that were dominant in undisturbed stands, where N. parvum was not found. Furthermore, N. parvum populations from trees planted in urban environments were more genetically diverse than populations from human disturbed but naturally regenerated stands of S. cordatum, indicating structure correlated with level of disturbance. It signifies a possible influence of human activity on the composition of fungal pathogens on a native host such as S. cordatum, and supports the hypothesis that, as with other organisms, generalist species are better invaders in human-disturbed environments.

Isolates of $N$. paruum were assigned to three subpopulations by the STRUCTURE analysis, based on allele frequencies across the loci, with very low or no admixture among the groups. This indicates that the N. parvum population associated with native S. cordatum in South Africa is a mixture of at least three independent sources, with different ancestral origins. The origin of the three groups is, however, unclear, because they could not be assigned to specific geographic regions or hosts in this study. Furthermore, N. parvum populations from trees planted in urban environments were more genetically diverse than populations from disturbed stands of S. cordatum among plantations of non-native Eucalyptus, indicating biological invasion with multiple introductions as a possible scenario. 

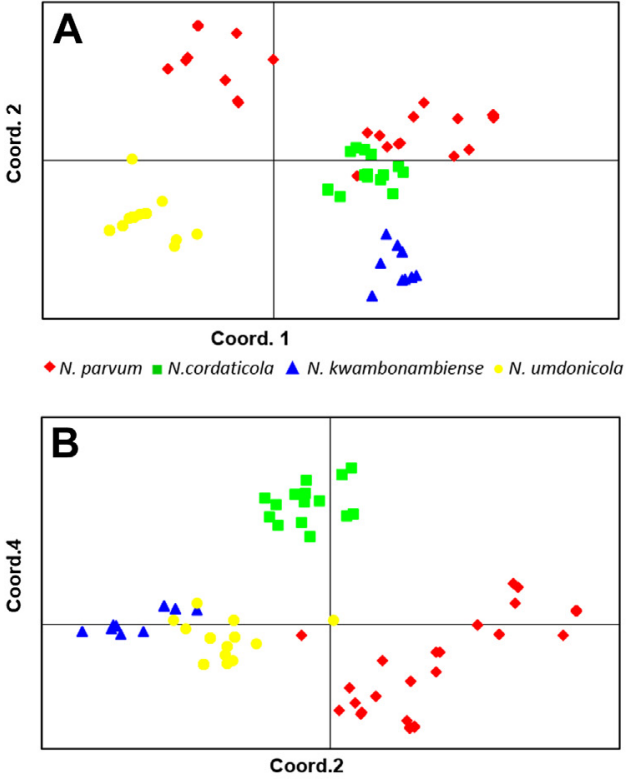

- N. parvum $=$ N.cordaticola $\Delta$ N. kwambonambiense - N. umdonicola

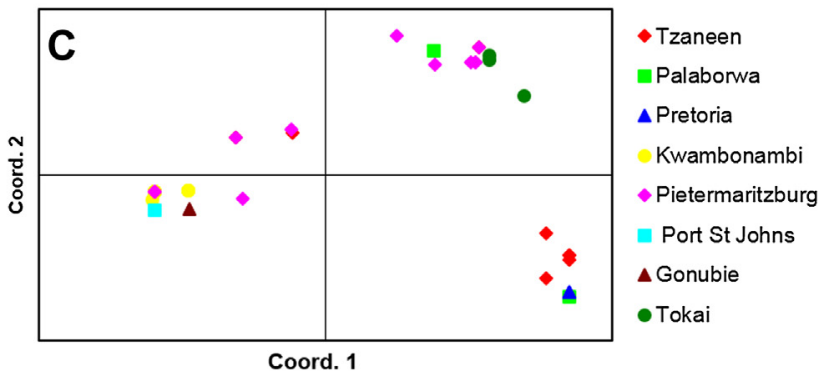

Fig 3 - Principal coordinate analyses (PCoA) of multilocus genotypes diversity for the 61 genotypes representing four different species, $\mathbf{N}$. cordaticola, $\mathbf{N}$. kwambonambiense, $\mathbf{N}$. umdonicola and $N$. parvum, analyzed with seven microsatellite markers (A, B) and the twenty-three genotypes of $N$. parvum from S. cordatum in South Africa representing 48 isolates of $N$. parvum (C). The grouping of isolates is in accordance with clusters identified by STRUCTURE analyses (Fig 2A), indicating four species and three sub-populations within $\mathrm{N}$. parvum.

The existence of numerous isolates with identical multilocus haplotypes, in all the different species and in particular in different populations of N. parvum, suggests that either asexual reproduction or a homothallic sexual cycle plays an important role in structuring these populations (Taylor et al., 1999; McDonald and Linde, 2002). Although Neofusicoccum species produce both sexual and asexual structures in their life cycle, these species are most commonly encountered in the asexual state, which might suggest that asexual reproduction is the main cause of the identical haplotypes. Sexual structures are known for N. parvum, but they have not been identified for $N$. cordaticola, N. kwambonambiense and $N$. umdonicola. There have been no studies considering whether they are exclusively outcrossing or alternatively, whether they can also reproduce homothallically. Since mode of reproduction plays an important role in population structure and diversity, future studies should interrogate this question. The

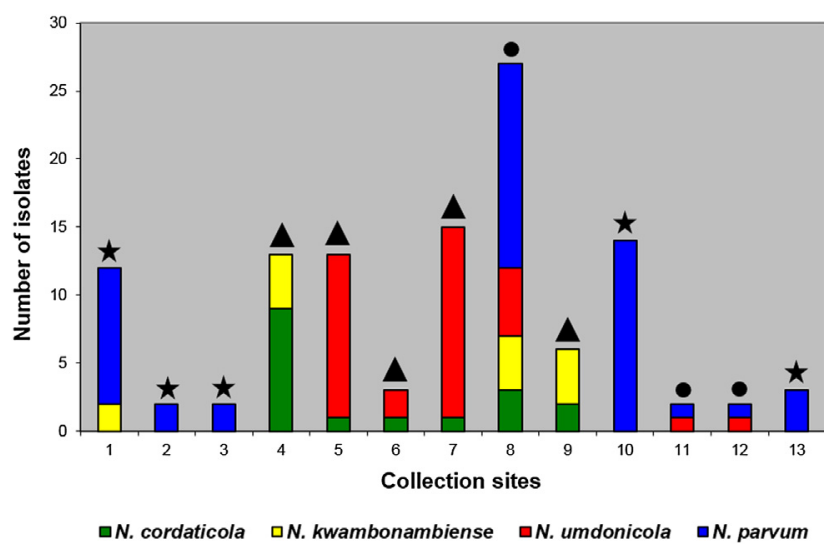

Fig 4 - Bars representing the distribution of four species from the Neofusicoccum parvum/ribis complex on Syzygium cordatum in the thirteen collection sites. Tzaneen (1P), Palaborwa (2P), Pretoria (3P), Sabie (4UD), Kosi Bay (5NU), Mkhuze (6UD), Sodwana Bay (7UD), Kwambonambi (8HD), Richards Bay (9UD), Pietermaritzburg (10P), Port St. Johns (11HD), Gonubie (12HD), Tokai (13P). P (O) = Isolates collected from planted trees in urban areas; UD $(\Delta)=$ Isolates collected from trees in natural, undisturbed stands; HD $(\star)=$ Isolates collected from trees in natural, human disturbed stands.

relatively small population sizes did not allow us to further analyze such questions for these species. However, all four species analyzed in this study displayed significant linkage disequilibrium for all the pairwise comparisons. This suggests that asexual reproduction might be dominant in these fungi, as was recently suggested for Australian and South African populations of $N$. parvum from a variety of hosts (Sakalidis et al., 2013).

Distribution of the four species in the N. parvum/N. ribis complex across $\mathrm{S}$. cordatum displayed clear differences on trees in environments affected by human activity or those in isolated natural stands. In most areas studied, at least two Neofusicoccum species coexisted on S. cordatum, with one of the species typically dominant. For example, $N$. cordaticola was the dominant species in the Sabie area of the Mpumalanga Province. In contrast, this species was present only in low numbers in the KwaZulu-Natal Province, co-existing either with $\mathrm{N}$. kwambonambiense or N. umdonicola, and it was not present in any other area. The Sabie area is at high altitude and has a climate very different to other areas sampled, with a very different floral composition surrounding the collection site (Mucina and Rutherford, 2006). In contrast, N. umdonicola was the dominant species at the three isolated collection sites in the National Reserves in northern KwaZulu Natal, including Sodwana Bay, Kosi Bay and Mkhuze, which are at low altitude with a subtropical climate and surrounded by undisturbed native flora. The dominance of one species in a particular niche might be related to ecological specialization (Devictor et al., 2010) of each species to defined environmental conditions.

Neofusicoccum parvum is a known generalist that infests various woody hosts around the world (Pennycook and Samuels, 1985; Slippers and Wingfield, 2007; De Wet et al., 2008; Sakalidis et al., 2013). This was also the dominant 
species in the N. parvum/N. ribis complex isolated from S. cordatum in the present study. With the exception of two isolates of N. kwambonambiense, only N. parvum was ever found on planted S. cordatum in urban areas. In contrast, N. paruum was not found in the isolated natural stands of S. cordatum, even though the number of sampled trees from which isolates of the four Neofusicoccum spp. were recovered was much higher in the natural stands than in urban areas. This effect was despite the close geographical proximity and climatic similarity of regions where other Neofusicoccum spp. are common. The results suggest that N. paruum is not native on S. cordatum in this region and that it is spreading from some non-native hosts to native $S$. cordatum, rather than vice versa. The other three species in the N. parvum/N. ribis complex isolated in this study were recently recorded from other hosts and countries (Sakalidis et al., 2013). However, in South Africa S. cordatum is the only known host of $N$. cordaticola and N. umdonicola, while $N$. kwambonambiense also occurs on the native tree species Terminalia sericea (Sakalidis et al., 2013). The area of origin of these fungi thus remains difficult to determine.

The genotypic diversity among the isolates of $N$. parvum differed between the populations from $S$. cordatum from natural stands within Eucalyptus plantations in the Kwambonambi area and two populations from planted S. cordatum in urban areas (Pietermaritzburg and Tzaneen). A low level of genotypic diversity and dominance of one multilocus genotype was observed in the N. parvum population from the Kwambonambi area. This population was from naturally growing S. cordatum that remained amongst non-native Eucalyptus plantations, also residing in the Myrtaceae that dominate this area. Neofusicoccum parvum is the most common species of Botryosphaeriaceae found in non-native Eucalyptus plantations in South Africa (Slippers et al., 2004b; Maleme, 2008). Some of the genotypes identified in the Kwambonambi population from S. cordatum were identical to N. parvum genotypes identified on Eucalyptus in this area (Sakalidis et al., 2013). These results strongly support the hypothesis that this pathogen spreads between these two hosts and that proximity to non-native Eucalyptus shaped the population structure of N. parvum on S. cordatum in this area. Interestingly N. parvum is rare on Eucalyptus in the native range of the tree in Australia, and the fungus is most likely native on some other host and geographic origin, which has yet to be determined (Slippers et al., 2004b; Burgess et al., 2005; Sakalidis et al., 2013).

In contrast to the $N$. paruum sub-population one that comprises Kwambonambi population, two populations from planted S. cordatum trees in Pietermaritzburg and Tzaneen (sub-clusters two and three, respectively) exhibited high levels of genotypic diversity with some genotypes overlapping between them. This was despite the fact that these two sites are more than $600 \mathrm{~km}$ apart. Neofusicoccum parvum isolates having different genotypes, but with the same ancestral origin (as defined by STRUCTURE), were also found on planted S. cordatum in other areas distant from each other, such as Pretoria and Tokai. The S. cordatum trees in urban environments are surrounded by various known hosts of N. parvum, such as Eucalyptus, Vitis vinifera and Mangifera indica and many ornamental plants (Jacobs, 2002; Van Niekerk et al., 2004; Slippers et al., 2004b; Sakalidis et al., 2013), which could have provided a source of this fungus. Thus, multiple introductions through human activities and movement between hosts would be common in these areas and could have influenced the genetic diversity and population structure of N. parvum on the planted S. cordatum in urban areas. Such movement of pathogens, in particular generalists such as N. parvum, could result in a population continuum over large areas, where gene flow among isolates might serve to maintain similar populations even at distant locations (McDonald and Linde, 2002).

Introduction of plants into non-native areas, changes in land use and intensive forestation are some of the human activities that directly influence plant pathogen movement as well as interactions with their hosts. The patterns of distribution of N. paruum, N. cordaticola, N. kwambonambiense and N. umdonicola on S. cordatum in different areas, ranging from isolated natural stands to environments where they have been affected by human activity provides vivid examples of this influence. This study shows that focusing on a fungal plant pathogen in only one habitat, natural forest, plantation or an urban planting, could overlook the dynamic relationship involved in invasive migration. It also highlights the importance of characterizing cryptic and population diversity of plant pathogens where one seeks to understand these patterns. More importantly, it also suggests that some (cryptic) fungal species are better adapted to colonization of disturbed environments than others. As with other organisms, this might also make them better invaders (Desprez-Loustau et al., 2007; Lee and Gelembiuk, 2008) and more important pathogens on a variety of hosts over larger areas (Slippers and Wingfield, 2007). A more complete understanding of such phenomena would inform quarantine and management options for phytopathogenic fungi.

\section{Acknowledgments}

We thank the National Research Foundation (NRF), members of Tree Protection Co-operative Programme (TPCP), the THRIP initiative of the Department of Trade and Industry, and the Department of Science and Technology (DST)/NRF Centre of Excellence in Tree Health Biotechnology (CTHB), South Africa for financial support. We are grateful for the assistance of $\mathrm{Dr}$ Ilaria Germishuizen (Institute of Commercial Forestry Research (ICFR), Pietermaritzburg, South Africa) who provided the map. We also thank the two anonymous reviewers for their helpful comments and suggestions for improving the manuscript.

\section{References}

Arnaud-Haond, S., Belkhir, K., 2007. GENCLONE: a computer program to analyse genotypic data, test for clonality and describe spatial clonal organization. Molecular Ecology Notes 7, 15-17. 
Begoude, B.A.D., Slippers, B., Wingfield, M.J., et al., 2010. Botryosphaeriaceae associated with Terminalia catappa in Cameroon, South Africa and Madagascar. Mycological Progress 9, 101-123.

Belkhir, K., Borsa, P., Chikhi, L., et al., 2004. GENETIX 4.05, logiciel sous Windows pour la g'en 'etique des Populations. Laboratoire G'enome, Populations, Interactions. CNRS UMR 5000. Universit'e de Montpellier II, Montpellier (France).

Black, W.C., Krafsur, E.S., 1985. A FORTRAN program for the calculation and analysis of 2-locus linkage disequilibrium coefficients. Theoretical and Applied Genetics 70, 491-496.

Burgess, T.I., Barber, P.A., Hardy, GEStJ., 2005. Botryosphaeria spp. associated with eucalypts in Western Australia including description of Fusicoccum macroclavatum sp. nov. Australasian Plant Pathology 34, 557-567.

Crous, P.W., Slippers, B., Wingfield, M.J., Rheeder, J., Marasas, W.F.O., Philips, A.J.L., Alves, A., Burgess, T., Barber, P., Groenewald, J.Z., 2006. Phylogenetic lineages in the Botryosphaeriaceae. Studies in Mycology 55, 235-253.

Desprez-Loustau, M.-L., Robin, C., Bu'ee, M., Courtecuisse, R., Garbaye, J., Suffert, F., Sache, I., Rizzo, D.M., 2007. The fungal dimension of biological invasions. Trends in Ecology and Evolution 22, 472-480.

Devictor, V., Clavel, J., Julliard, R., Lavergne, S., Mouillot, D., Thuiller, W., Venail, P., et al., 2010. Defining and measuring ecological specialization. Journal of Applied Ecology 47, 15-25.

De Wet, J., Slippers, B., Preisig, O., et al., 2008. Phylogeny of the Botryosphaeriaceae reveals patterns of host association. Molecular Phylogenetics and Evolution 46, 116-126.

Earl, D.A., VonHoldt, B.M., 2012. STRUCTURE HARVESTER: a website and program for visualizing STRUCTURE output and implementing the Evanno method. Conservation Genetics Resources 4, 359-361.

Evanno, G., Regnaut, S., Goudet, J., 2005. Detecting the number of clusters of individuals using the software STRUCTURE: a simulation study. Molecular Ecology 14, 2611-2620.

Fisher, M.C., Henk, D., Briggs, C.J., et al., 2012. Emerging fungal threats to animal, plant and ecosystem health. Nature 484 (7393), 186-194.

Jacobs, R., 2002. Characterisation of Botryosphaeria Species from Mango in South Africa. MSc thesis. University of Pretoria.

Lee, C.E., Gelembiuk, G.W., 2008. Evolutionary origins of invasive populations. Evolutionary Applications 1 (3), 427-448.

McDonald, B.A., Linde, C., 2002. Pathogen population genetics, evolutionary potential, and durable resistance. Annual Review of Phytopathology 40, 349-379.

Maleme, H.M., 2008. Characterisation of Latent Botryosphaeriaceae on Diverse Eucalyptus Species. MSc thesis. University of Pretoria.

Mohali, R.S., Slippers, B., Wingfield, M.J., 2007. Identification of Botryosphaeriaceae from Eucalyptus, Acacia and Pinus in Venezuela. Fungal Diversity 25, 103-125.

Mucina, L., Rutherford, M.C. (Eds.), 2006. The Vegetation of South Africa, Lesotho and Swaziland. Strelitzia 19. South African National Biodiversity Institute, Pretoria.

Nei, M., 1978. Estimation of average heterozygosity and genetic distance from a small number of individuals. Genetics 89 , 583-590.

Palgrave, K.C., 1977. Trees of Southern Africa, first ed. C. Struik Publishers, South Africa.

Pavlic, D., Slippers, B., Coutinho, T.A., et al., 2007. Botryosphaeriaceae occurring on native Syzygium cordatum in South Africa and their potential threat to Eucalyptus. Plant Pathology 56, 624-636.

Pavlic, D., Slippers, B., Coutinho, T.A., et al., 2009a. Multiple gene genealogies and phenotypic data reveal cryptic species of the
Botryosphaeriaceae: a case study on the Neofusicoccum parvum/N. ribis complex. Molecular Phylogenetics and Evolution 51, 259-268.

Pavlic, D., Slippers, B., Coutinho, T.A., et al., 2009b. Molecular and phenotypic characterisation of three phylogenetic species discovered within the Neofusicoccum parvum/N. ribis complex. Mycologia 101, 636-647.

Peakall, R., Smouse, P.E., 2006. Genalex version 6: genetic analyses in Excel. Population genetic software for teaching and research. Molecular Ecology Notes 6, 288-295.

Peakall, R., Smouse, P.E., 2012. Genalex version 6.5: genetic analysis in Excel. Population genetic software for teaching and research-an update. Bioinformatics 28, 2537-2539.

Pennycook, S.R., Samuels, G.J., 1985. Botryosphaeria and Fusicoccum species associated with ripe fruit rot of Actinidia deliciosa (Kiwifruit) in New Zealand. Mycotaxon 24, 445-458.

Pritchard, J.K., Stephens, M., Donnelly, P., 2000. Inference of population structure using multilocus genotype data. Genetics 155, 945-959.

Rousset, F., 1997. Genetic differentiation and estimation of gene flow from F-statistics under isolation by distance. Genetics 145 , 1219-1228.

Sakalidis, M.L., GEStJ, Hardy, Burgess, T.I., 2011. Use of the Genealogical Sorting Index (GSI) to delineate species boundaries in the Neofusicoccum parvum-Neofusicoccum ribis species complex. Molecular Phylogenetics and Evolution 60, 333-344.

Sakalidis, M.L., Slippers, B., Wingfield, B.D., et al., 2013. The challenge of understanding the origin, pathways and extent of fungal invasions: global populations of the Neofusicoccum parvum-N. ribis species complex. Diversity and Distributions. http://dx.doi.org/10.1111/ddi.12030.

Santini, A., Ghelardini, L., De Pace, C., et al., 2013. Biogeographical patterns and determinants of invasion by forest pathogens in Europe. New Phytologist 197, 238-250.

Slippers, B., Burgess, T., Crous, P.W., et al., 2004a. Development of SSR markers for Botryosphaeria spp. with Fusicoccum anamorphs. Molecular Ecology Notes 4, 675-677.

Slippers, B., Fourie, G., Crous, P.W., et al., 2004b. Speciation and distribution of Botryosphaeria spp. on native and introduced Eucalyptus trees in Australia and South Africa. Studies in Mycology 50, 343-358.

Slippers, B., Smit, W.A., Crous, P.W., et al., 2007. Taxonomy, phylogeny and identification of Botryosphaeriaceae associated with pome and stone fruit trees in South Africa and other regions of the world. Plant Pathology 56, 128-139.

Slippers, B., Summerell, B.A., Crous, P.W., et al., 2005. Preliminary studies on Botryosphaeria species from Wollemia nobilis and related southern hemisphere conifers in Australasia and South Africa. Australasian Plant Pathology 34, 213-220.

Slippers, B., Wingfield, M.J., 2007. Botryosphaeriaceae as endophytes and latent pathogens of woody plants: diversity, ecology and impact. Fungal Biology Reviews 21, 90-106.

Szpiech, Z.A., Jakobsson, M., Rosenberg, N.A., 2008. ADZE: a rarefaction approach for counting alleles private to combinations of populations. Bioinformatics 24, 2498-2504.

Taylor, J.W., Jacobson, D.J., Fisher, M.C., 1999. The evolution of asexual fungi: Reproduction, Speciation and Classification. Annual Review of Phytopathology 37, 197-246.

Van Niekerk, Crous, P., Groenewald, J.Z., et al., 2004. DNA phylogeny, morphology and pathogenicity of Botryosphaeria species on grapevines. Mycologia 96, 781-798.

Yeh, F.C., Yang, R.-C., Boyle, T., 1999. Popgene: Microsoft Window-based Freeware for population Genetic Analysis, Version 1.31. University of Alberta, Canada. Available at: URL: http://www.ualberta.ca/ fyeh/download.htm. 


\section{SUPPLEMENTARY SECTION}

Table 1 Isolates analysed in this study

\begin{tabular}{|c|c|c|c|c|c|c|}
\hline $\begin{array}{c}\text { Culture } \\
\text { no. }{ }^{1,2}\end{array}$ & $\begin{array}{l}\text { Other } \\
\text { no. }^{3}\end{array}$ & $\begin{array}{l}\text { Tree } \\
\text { no. }^{4} \\
\end{array}$ & Identity & Geographic origin $^{5}$ & $\begin{array}{l}\text { Haplotype } \\
\text { locations }\end{array}$ & $\begin{array}{l}\text { Haplotype } \\
\text { host }^{7}\end{array}$ \\
\hline CMW 14128 & & 1 & Neofusicoccum parvum & Tzaneen, Northern Province & TZ1 & S1 \\
\hline CMW 14129 & & 2 & N. parvum & Tzaneen, Northern Province & $\mathrm{TZ2}$ & S2 \\
\hline CMW 14130 & & 3 & N. parvum & Tzaneen, Northern Province & $\mathrm{TZ3}$ & S3 \\
\hline CMW 14133 & & 4 & N. parvum & Tzaneen, Northern Province & TZ4 & S4 \\
\hline CMW 14134 & & 5 & N. parvum & Tzaneen, Northern Province & TZ5 & S5 \\
\hline CMW 14135 & & 6 & N. parvum & Tzaneen, Northern Province & TZ6 & S6 \\
\hline CMW 14136 & & 7 & N. kwambonamniense & Tzaneen, Northern Province & TZ7 & S7 \\
\hline CMW 14137 & & 8 & N. parvum & Tzaneen, Northern Province & TZ8 & S8 \\
\hline CMW 14138 & & 9 & N. parvum & Tzaneen, Northern Province & TZ3 & S3 \\
\hline CMW 14139 & & 10 & N. parvum & Tzaneen, Northern Province & TZ9 & S9 \\
\hline CMW 14140 & CBS 123641 & 11 & N. kwambonamniense & Tzaneen, Northern Province & TZ10 & S10 \\
\hline CMW 14141 & & 11 & N. parvum & Tzaneen, Northern Province & TZ5 & S5 \\
\hline CMW 14142 & & 1 & N. parvum & Phalaborwa, Northern Province & PB1 & S3 \\
\hline CMW 14143 & & 1 & N. parvum & Phalaborwa, Northern Province & PB2 & S11 \\
\hline CMW 14144 & & 1 & N. cordaticola & Sabie, Mpumalanga Province & K1 & S12 \\
\hline CMW 14145 & & 1 & N. cordaticola & Sabie, Mpumalanga Province & $\mathrm{K} 2$ & $\mathrm{~S} 13$ \\
\hline CMW 14146 & & 1 & N. cordaticola & Sabie, Mpumalanga Province & K3 & S14 \\
\hline CMW 14147 & & 1 & N. cordaticola & Sabie, Mpumalanga Province & K2 & S13 \\
\hline CMW 14148 & & 2 & N. cordaticola & Sabie, Mpumalanga Province & K4 & S15 \\
\hline CMW 14149 & & 2 & N. cordaticola & Sabie, Mpumalanga Province & K5 & S16 \\
\hline CMW 14150 & & 2 & N. cordaticola & Sabie, Mpumalanga Province & K2 & S13 \\
\hline CMW 14151 & CBS 123637 & 3 & N. cordaticola & Sabie, Mpumalanga Province & K6 & S17 \\
\hline CMW 14152 & & 3 & N. cordaticola & Sabie, Mpumalanga Province & K7 & S18 \\
\hline CMW 14153 & & 4 & N. kwambonamniense & Sabie, Mpumalanga Province & K8 & S10 \\
\hline CMW 14154 & & 4 & N. kwambonamniense & Sabie, Mpumalanga Province & K9 & S19 \\
\hline CMW 14155 & CBS 123642 & 5 & N. kwambonamniense & Sabie, Mpumalanga Province & K10 & S7 \\
\hline CMW 14156 & & 5 & N. kwambonamniense & Sabie, Mpumalanga Province & K10 & S7 \\
\hline CMW 27901 & & 1 & N. parvum & Pretoria, Gauteng Province & P1 & S20 \\
\hline CMW 29125 & & 1 & N. parvum & Pretoria, Gauteng Province & P1 & S20 \\
\hline CMW 14055 & & 1 & N. umdonicola & Kosi Bay, KwaZulu Natal Province & M1 & S21 \\
\hline CMW 14056 & CBS 123635 & 1 & N. cordaticola & Kosi Bay, KwaZulu Natal Province & M2 & S22 \\
\hline CMW 14057 & & 2 & N. umdonicola & Kosi Bay, KwaZulu Natal Province & M3 & S23 \\
\hline CMW 14058 & CBS 123645 & 3 & N. umdonicola & Kosi Bay, KwaZulu Natal Province & M4 & S24 \\
\hline CMW 14059 & & 4 & N. umdonicola & Kosi Bay, KwaZulu Natal Province & M5 & S25 \\
\hline CMW 14060 & CBS123646 & 5 & N. umdonicola & Kosi Bay, KwaZulu Natal Province & M6 & S26 \\
\hline CMW 14061 & & 6 & N. umdonicola & Kosi Bay, KwaZulu Natal Province & M7 & S27 \\
\hline CMW 14062 & & 7 & N. umdonicola & Kosi Bay, KwaZulu Natal Province & M3 & S23 \\
\hline CMW 14068 & & 8 & N. umdonicola & Kosi Bay, KwaZulu Natal Province & M5 & S25 \\
\hline CMW 14098 & & 9 & N. umdonicola & Kosi Bay, KwaZulu Natal Province & M8 & S28 \\
\hline CMW 14099 & & 10 & N. umdonicola & Kosi Bay, KwaZulu Natal Province & M9 & S29 \\
\hline CMW 14100 & & 11 & N. umdonicola & Kosi Bay, KwaZulu Natal Province & M10 & S30 \\
\hline CMW 14101 & & 12 & N. umdonicola & Kosi Bay, KwaZulu Natal Province & M3 & S23 \\
\hline CMW 14047 & & 1 & N. umdonicola & Mkuze, KwaZulu Natal Province & $\mathrm{CF} 1$ & S31 \\
\hline CMW 14051 & & 2 & N. umdonicola & Mkuze, KwaZulu Natal Province & $\mathrm{CF} 2$ & S27 \\
\hline CMW 14054 & CBS 123636 & 3 & N. cordaticola & Mkuze, KwaZulu Natal Province & $\mathrm{CF} 3$ & S32 \\
\hline CMW 13990 & & 1 & N. umdonicola & Sodwana Bay, KwaZulu Natal Province & SW1 & S30 \\
\hline CMW 13991 & & 1 & N. umdonicola & Sodwana Bay, KwaZulu Natal Province & SW1 & S30 \\
\hline CMW 13992 & CBS 123634 & 1 & N. cordaticola & Sodwana Bay, KwaZulu Natal Province & SW2 & S33 \\
\hline CMW 13993 & & 2 & N. umdonicola & Sodwana Bay, KwaZulu Natal Province & SW1 & S30 \\
\hline CMW 13994 & & 2 & N. umdonicola & Sodwana Bay, KwaZulu Natal Province & SW3 & S25 \\
\hline CMW 13995 & & 2 & N. umdonicola & Sodwana Bay, KwaZulu Natal Province & SW3 & S25 \\
\hline CMW 13996 & & 2 & N. umdonicola & Sodwana Bay, KwaZulu Natal Province & SW4 & S27 \\
\hline CMW 13997 & & 2 & N. umdonicola & Sodwana Bay, KwaZulu Natal Province & SW5 & S34 \\
\hline CMW 14006 & & 3 & N. umdonicola & Sodwana Bay, KwaZulu Natal Province & SW4 & S27 \\
\hline CMW 14007 & & 3 & N. umdonicola & Sodwana Bay, KwaZulu Natal Province & SW6 & S35 \\
\hline CMW 14008 & & 4 & N. umdonicola & Sodwana Bay, KwaZulu Natal Province & SW7 & S28 \\
\hline CMW 14106 & CBS 123644 & 4 & N. umdonicola & Sodwana Bay, KwaZulu Natal Province & SW8 & S36 \\
\hline CMW 14010 & & 5 & N. umdonicola & Sodwana Bay, KwaZulu Natal Province & SW7 & S28 \\
\hline CMW 14011 & & 5 & N. umdonicola & Sodwana Bay, KwaZulu Natal Province & SW9 & S26 \\
\hline CMW 14012 & & 6 & N. umdonicola & Sodwana Bay, KwaZulu Natal Province & SW4 & S27 \\
\hline
\end{tabular}


Table 1 Continued

\begin{tabular}{|c|c|c|c|c|c|c|}
\hline $\begin{array}{c}\text { Culture } \\
\text { no. }{ }^{1,2}\end{array}$ & $\begin{array}{l}\text { Other } \\
\text { no. }^{3}\end{array}$ & $\begin{array}{l}\text { Tree } \\
\text { no. } 4\end{array}$ & Identity & Geographic origin $^{5}$ & $\begin{array}{l}\text { Haplotype } \\
\text { locations }\end{array}$ & $\begin{array}{l}\text { Haplotype } \\
\text { host } 7\end{array}$ \\
\hline CMW 14016 & & 1 & N. umdonicola & Kwambonambi, KwaZulu Natal Province & KWM1 & $\mathrm{S} 25$ \\
\hline CMW 14018 & & 2 & N. parvum & Kwambonambi, KwaZulu Natal Province & KWM2 & S37 \\
\hline CMW 14019 & & 2 & N. parvum & Kwambonambi, KwaZulu Natal Province & KWM3 & S38 \\
\hline CMW 14020 & & 2 & N. parvum & Kwambonambi, KwaZulu Natal Province & KWM4 & S39 \\
\hline CMW 14021 & & 3 & N. parvum & Kwambonambi, KwaZulu Natal Province & KWM2 & S37 \\
\hline CMW 14022 & & 4 & N. parvum & Kwambonambi, KwaZulu Natal Province & KWM2 & S37 \\
\hline CMW 14023 & CBS 123639 & 5 & N. kwambonamniense & Kwambonambi, KwaZulu Natal Province & KWM5 & $\mathrm{S} 40$ \\
\hline CMW 14024 & CBS 123638 & 6 & N. parvum & Kwambonambi, KwaZulu Natal Province & KWM2 & S37 \\
\hline CMW 14025 & CBS 123640 & 7 & N. kwambonamniense & Kwambonambi, KwaZulu Natal Province & KWM6 & S41 \\
\hline CMW 14027 & & 7 & N. parvum & Kwambonambi, KwaZulu Natal Province & KWM2 & S37 \\
\hline CMW 14028 & & 8 & N. umdonicola & Kwambonambi, KwaZulu Natal Province & KWM7 & S27 \\
\hline CMW 14030 & & 9 & N. parvum & Kwambonambi, KwaZulu Natal Province & KWM8 & $\mathrm{S} 42$ \\
\hline CMW 14031 & & 10 & N. kwambonamniense & Kwambonambi, KwaZulu Natal Province & KWM9 & $\mathrm{S} 43$ \\
\hline CMW 14032 & & 11 & N. parvum & Kwambonambi, KwaZulu Natal Province & KWM2 & S37 \\
\hline CMW 14035 & & 11 & N. cordaticola & Kwambonambi, KwaZulu Natal Province & KWM10 & S44 \\
\hline CMW 14036 & & 12 & N. parvum & Kwambonambi, KwaZulu Natal Province & KWM2 & S37 \\
\hline CMW 14037 & & 12 & N. parvum & Kwambonambi, KwaZulu Natal Province & KWM3 & $\mathrm{S} 38$ \\
\hline CMW 14038 & & 12 & N. parvum & Kwambonambi, KwaZulu Natal Province & KWM3 & $\mathrm{S} 38$ \\
\hline CMW 14039 & & 12 & N. parvum & Kwambonambi, KwaZulu Natal Province & KWM2 & S37 \\
\hline CMW 14040 & & 13 & N. parvum & Kwambonambi, KwaZulu Natal Province & KWM2 & S37 \\
\hline CMW 14041 & & 14 & N. cordaticola & Kwambonambi, KwaZulu Natal Province & KWM11 & $\mathrm{S} 45$ \\
\hline CMW 14042 & & 14 & N. cordaticola & Kwambonambi, KwaZulu Natal Province & KWM12 & S46 \\
\hline CMW 14045 & & 15 & N. parvum & Kwambonambi, KwaZulu Natal Province & KWM2 & S37 \\
\hline CMW 14046 & & 16 & N. kwambonamniense & Kwambonambi, KwaZulu Natal Province & KWM5 & $\mathrm{S} 40$ \\
\hline CMW 14125 & & 17 & N. umdonicola & Kwambonambi, KwaZulu Natal Province & KWM7 & S27 \\
\hline CMW 14126 & & 17 & N. umdonicola & Kwambonambi, KwaZulu Natal Province & KWM7 & S27 \\
\hline CMW 14127 & CBS 123648 & 17 & N. umdonicola & Kwambonambi, KwaZulu Natal Province & KWM13 & S47 \\
\hline CMW 14119 & & 1 & N. kwambonamniense & Richards Bay, KwaZulu Natal Province & $\mathrm{R} 1$ & $\mathrm{~S} 48$ \\
\hline CMW 14120 & & 1 & N. kwambonamniense & Richards Bay, KwaZulu Natal Province & R 1 & $\mathrm{~S} 48$ \\
\hline CMW 14121 & & 1 & N. kwambonamniense & Richards Bay, KwaZulu Natal Province & $\mathrm{R} 2$ & S49 \\
\hline CMW 14122 & & 2 & N. cordaticola & Richards Bay, KwaZulu Natal Province & R 3 & $\mathrm{~S} 50$ \\
\hline CMW 14123 & CBS 123643 & 3 & N. kwambonamniense & Richards Bay, KwaZulu Natal Province & $\mathrm{R} 4$ & S51 \\
\hline CMW 14124 & & 3 & N. cordaticola & Richards Bay, KwaZulu Natal Province & R 5 & S52 \\
\hline CMW 14081 & & 1 & N. parvum & Pietermaritzburg, KwaZulu Natal Pravince & PM1 & S37 \\
\hline CMW 14082 & & 1 & N. parvum & Pietermaritzburg, KwaZulu Natal Pravince & PM2 & S9 \\
\hline CMW 14084 & & 2 & N. parvum & Pietermaritzburg, KwaZulu Natal Pravince & PM1 & S37 \\
\hline CMW 14085 & & 3 & N. parvum & Pietermaritzburg, KwaZulu Natal Pravince & PM3 & S53 \\
\hline CMW 14086 & & 3 & N. parvum & Pietermaritzburg, KwaZulu Natal Pravince & PM4 & $\mathrm{S} 2$ \\
\hline CMW 14087 & & 3 & N. parvum & Pietermaritzburg, KwaZulu Natal Pravince & PM5 & S54 \\
\hline CMW 14088 & & 3 & N. parvum & Pietermaritzburg, KwaZulu Natal Pravince & PM6 & S55 \\
\hline CMW 14089 & & 4 & N. parvum & Pietermaritzburg, KwaZulu Natal Pravince & PM7 & S56 \\
\hline CMW 14090 & & 4 & N. parvum & Pietermaritzburg, KwaZulu Natal Pravince & PM1 & S37 \\
\hline CMW 14091 & & 4 & N. parvum & Pietermaritzburg, KwaZulu Natal Pravince & PM8 & $\mathrm{S} 1$ \\
\hline CMW 14092 & & 5 & N. parvum & Pietermaritzburg, KwaZulu Natal Pravince & PM1 & S37 \\
\hline CMW 14093 & & 6 & N. parvum & Pietermaritzburg, KwaZulu Natal Pravince & PM7 & S56 \\
\hline CMW 14094 & & 6 & N. parvum & Pietermaritzburg, KwaZulu Natal Pravince & PM9 & S57 \\
\hline CMW 14095 & & 7 & N. parvum & Pietermaritzburg, KwaZulu Natal Pravince & PM1 & S37 \\
\hline CMW 14096 & & 1 & N. umdonicola & Port St Johns, Eastern Cape Province & SJP1 & $\mathrm{S} 27$ \\
\hline CMW 14097 & & 1 & N. parvum & Port St Johns, Eastern Cape Province & SJP2 & S39 \\
\hline CMW 14079 & CBS 123647 & 1 & N. umdonicola & Gonubie, Eastern Cape Province & G1 & $\mathrm{S} 35$ \\
\hline CMW 14080 & CBS123651 & 1 & N. parvum & Gonubie, Eastern Cape Province & $\mathrm{G} 2$ & S58 \\
\hline CMW 14110 & & 1 & N. parvum & Tokai, Western Cape Province & $\mathrm{T} 1$ & S59 \\
\hline CMW 14111 & & 1 & N. parvum & Tokai, Western Cape Province & $\mathrm{T} 2$ & S60 \\
\hline CMW 14112 & & 1 & N. parvum & Tokai, Western Cape Province & $\mathrm{T} 3$ & S61 \\
\hline
\end{tabular}

1,3 Abbreviations of culture collections: CMW = Tree Protection Co-operative Programme, Forestry and

Agricultural Biotechnology Institute, University of Pretoria, South Africa; CBS = Centraalbureau voor

Schimmelcultures Utrecht, Netherlands

${ }^{2}$ Isolates identified in this study are in boldface. 
${ }^{4}$ Tree numbers from which isolates were collected at each of sites

${ }^{5}$ All isolates were collected from $S$. cordatum trees in different provinces of South Africa by D. Pavlic-Zupanc

${ }^{6}$ Different haplotypes identified in each location

${ }^{7}$ Different haplotypes (S1-S61) identified among all 114 isolates from S. cordatum trees 